\title{
An empirical analysis of factors influencing customer responsiveness to mobile advertising
}

Received (in revised form): 29th April, 2008

\section{Monika Mital}

has 15 years of experience, out of which ten years is in teaching. She is currently a faculty in the Information Systems Group of a reputed Business School in India, Jaipuria Institute of Management, Lucknow. Her research interests include themes on Human Computer Interaction, specifically e-learning, mobile advertising and internet business, etc. She has to her credit approximately ten papers in reputed and indexed international journals and a book, Management Information Systems, published by Oxford University Press, India.

Keywords mobile marketing, consumer acceptance, mobile advertising, contextualisation of mobile marketing, intrusiveness of mobile marketing

Abstract When looking at marketing communication from a consumer perspective, the issue of media effectiveness is challenging. Many consumers have attitudes, aspirations and purchasing patterns that are different in mobile marketing compared to what companies have been used to. The primary objective of this research study is to gain an insight into the perception of mobile users, towards mobile advertising and their utility value in terms of impact on the purchase decision. Finally, an attempt has also been made to broadly concretize some features enhancing the acceptability/utility of mobile advertisements, based on user characteristics like age, work schedule, mobile usage, etc. Journal of Database Marketing \& Customer Strategy Management (2008) 15, 119-125.

doi:10.1057/dbm.2008.6

\section{INTRODUCTION}

Innovation creates marketing opportunities and challenges. Mobile advertising, which is an area of mobile commerce, is a form of advertising that targets users of handheld wireless devices such as mobile phones and PDAs. In comparison with traditional advertising, the main advantages of mobile advertising are: personal touch, tailor-made to suit individuals, and hence can be considered cost-effective. Mobile advertising can reach the target customers anywhere anytime. In order to promote the selling of products or services, all the activities required to communicate with the customers are transferred through mobile devices. Combining with the customer's user profile and context situation, advertising companies can provide the target customers exactly the advertisement information they desire, not just 'spam' them with advertisements they are not interested in.

Mobile media, for example, transcend traditional communication and support one-to one, many-to-many and mass communication. Phones and personal digital assistants increase the availability, frequency, and speed of communication. Yet the technology associated with these devices, which let marketers personally communicate with consumers, continues to evolve. The most popular mobile application is referred to as text messaging or short message service (SMS). Studies on this new 
advertising medium indicate that mobile advertising campaigns can generate responses that are as high as 40 per cent, compared with a 3 per cent response rate through direct mail and 1 per cent with internet banner ads. ${ }^{1}$

The high diffusion of SMS facilitates analysing usage behaviour and hints at the commercial potential of future communication services. Multimedia messaging services (MMS), for example, will build on the success of SMS but allow for richer content based on similar asynchronous, digital, and interactive communication. Studying interactive mobile services such as SMS and MMS suggests drawing upon theories in marketing, consumer behaviour, psychology, and adoption to investigate their organisational and personal use.

In traditional research concerning advertising and media effects, the consumer's perspective has been largely overlooked. Traditional marketing communication planning has focused on the marketer's objectives of reaching relevant consumers. ${ }^{2}$ An open question is, however, what reaching means when considering the empowered and active consumer. A key issue is the responsiveness of the consumer to marketing communication.

Responsiveness depicts the consumer's willingness to receive and respond to marketing communication and can be viewed as a function of the content and the context of the message. Any channel can and should be evaluated according to consumer responsiveness in order to understand communication effects and effectiveness. Consumer responsiveness is potentially more effective than permission because it assumes consumer attention rather than merely permission.

\section{OBJECTIVES OF THE STUDY}

The primary objective of this research study is to gain an insight into the perception of mobile users towards mobile advertising and their utility value in terms of impact on the purchase decision. Hence, we are attempting to explore consumers' responsiveness to mobile marketing, taking into cognizance the impact of demographic factors like age, gender, occupation, etc. From the marketer's perspective it is crucial to know the utility of mobile advertising, as far as having an impact on purchase decision of a customer is concerned. From an academic perspective the issue of responsiveness to a media is interesting in itself. The media in use influences the effect of the marketing communication in addition to the marketing message. Finally, an attempt has also been made to broadly concretise some features enhancing the acceptability/utility of mobile advertisements.

\section{LITERATURE REVIEW}

Countries such as Japan, New Zealand, Germany and the UK, which have cost-effective and interoperable wireless structures, a high penetration of mobile phones, and a relatively low cost for the SMS messaging service, have experienced remarkable success with the SMS application. ${ }^{3}$ However, Heinonen and Strandvik ${ }^{4}$ showed that mobile channels are perceived to be more personal than traditional and e-mail channels. This creates high expectations for the relevance of marketing communication messages. Consumers expect messages to be personal and of high interest and this make the disappointment greater when they get undesired messages. Mobile advertising may even step over the line of discretion and invade consumers' privacy because of the personal nature of the mobile device. For example, unsolicited e-mail and spam mail are considered an invasion of privacy and have actually become a serious problem for many consumers. ${ }^{5}$ Li et al. ${ }^{6}$ discusses how negative reactions like irritation arise through intrusion advertising. The channel influences consumer responsiveness to marketing communication by being perceived as either disturbing or acceptable. ${ }^{7}$ 
If the consumer considers marketing communication via a channel as disturbing, it may negatively affect the attention to and perception of the message. In contrast, the channel may also enhance the acceptance of the marketing communication if it is perceived as appropriate for the specific marketing communication. Also, some consumers may perceive the channels as neutral, neither disturbing nor accepted.

New media in the digital economy have created potentially powerful tools for direct and interactive marketing. Traditional marketing communication strategies have been based on the interruption logic, where the consumer is forced to momentarily pay attention. Permission marketing was introduced as a new managerial approach in marketing communication. It has been argued that firms benefit from getting consumers' permission to be contacted. ${ }^{8}$ Permission from the consumer would resolve the difficulties to get access to the consumer. Permission is, however, not necessarily a guarantee that the consumer pays attention; it is only a door opener and gives an indication of the consumer's potential interest areas.

One of the main challenges and opportunities for mobile advertising companies is to understand and respect the personal nature of the usage of mobile phones. ${ }^{1,3,4,9}$ The key is to use interactive wireless media to provide customers with time- and location-sensitive, personalised information that promotes goods, services, and ideas, thereby generating value for all stakeholders. ${ }^{10}$ The mobile advertising relevance can be influenced by the contextualisation of advertising messages. ${ }^{11,12}$ Barwise and Strong ${ }^{9}$ take up the flexibility of mobile advertising and its time-based nature, but also the fact that the small screens restrict the length of the message. Barnes stresses the interactive nature of mobile advertising and the ability to use contextual information to target the messages to individual receivers, in other words to personalise the message. ${ }^{13}$ Location-aware advertising messages are creating 5-10 times higher click-through rates compared to traditional internet advertising messages. ${ }^{14}$

\section{METHODOLOGY}

The research design chosen for the study is exploratory and conclusive. A survey instrument was developed based on previous studies done on consumer perceptions of mobile advertising. Quota sampling has been employed with the questionnaires being sent to approximately 1,000 respondents. However, 865 questionnaires were found complete in all respects. An attempt has been made to keep the sample fairly representative across various occupations and age groups. The response rate was 86.5 per cent.

Almost 54 per cent of the respondents belonged to the 20-30-year-old age group and approximately 30 per cent of the respondents belonged to the age group of more than 30 years. Thirty per cent of the respondents were students, 27 per cent were in service, 14 per cent were housewives, and 29 per cent were in business. Nearly 60 per cent were males and 40.3 per cent were females. Almost 85 per cent of the respondents rated their usage of mobile in the range of medium to high. Seventy-five per cent of the respondents used their mobiles primarily for personal communication.

The study used a Likert scale of 1-5 to elicit response of mobile users (ie 1 for totally disagree and 5 for totally agree). The statistical techniques used to analyse the data are factor analysis to factorise the perception of mobile users towards mobile advertising and chi-square analysis to evaluate the impact of mobile advertising on purchase/brand decision and brand recall.

\section{RESULTS}

The factor analysis results are shown in Table $1-3$. The variance explained by the 
initial solution, extracted components, and the rotated components is displayed in Table 1. The total variance shown in Table 1 accounted for by each of the six components explains nearly 71 per cent of the variability in the original 13 variables. So we can reduce the original data set by using these three components (eigen values greater than 1 as shown in Table 1) with only 29 per cent loss of information.

Looking at Table 3 , the rotated factor matrix, and the study shows three factors (which represent the three perceptual dimensions about mobile advertising) derived from 13 variables (which represent

Table 1: Total variance explained

\begin{tabular}{llll}
\hline Component & \multicolumn{3}{l}{ Rotation sums of squared loadings } \\
\cline { 2 - 4 } & Total & $\begin{array}{c}\text { \% of } \\
\text { variance }\end{array}$ & Cumulative \% \\
\hline 1 & 2.572 & 29.781 & 29.781 \\
2 & 2.429 & 23.685 & 53.466 \\
3 & 1.891 & 17.550 & 71.016 \\
\hline
\end{tabular}

Table 2: KMO and Bartlett's test

\begin{tabular}{ll}
\hline $\begin{array}{l}\text { Kaiser-Meyer-Olkin measure of } \\
\text { sampling adequacy }\end{array}$ & 0.820 \\
$\begin{array}{l}\text { Bartlett's test of approx. chi-square } \\
\text { sphericity }\end{array}$ & 1575.073 \\
d.f. & 78 \\
Sig. & 0.000 \\
\hline
\end{tabular}

the perception of mobile users towards mobile advertising). The components of each factor are shown highlighted in Table 3.

The factor analysis identified 13 items in three groups, as Factor 1, Factor 2, and Factor 3, and the Cronbach reliability alpha coefficient for the 13-item scale was 0.703. We then investigated further optimisation of the instrument by examining the reliability coefficient of each factor independently. We then found that the four-item Factor 1 had a reliability coefficient of 0.788 , fiveitem Factor 2 had a reliability coefficient of 0.811, and four-item Factor 3 had a reliability coefficient of 0.834 .

Looking at Table 3, we find that the variables: mobile ads being less informative, not suiting personal needs, inappropriate timing, and clutter are loaded to Factor 1 and since all the factors are related to lack of contextualisation and personalisation, this factor can be labelled 'Lack of Contextualisation'.

Factor 2 has components brand recall, recall of sales/promotion information, recall of product/services, ad positioning, repetitive nature of mobile ads, and positive impact of mobile ads. Since all these components are related to perceived usefulness of mobile advertising, this factor can be labelled 'Perceived Usefulness' of mobile ads.

Factor 3 has components disturbance at work, busy work schedule, wastage of time,

Table 3: Rotated component matrix

\begin{tabular}{lcrr}
\hline & \multicolumn{2}{l}{ Component } & $\mathbf{3}$ \\
\cline { 2 - 4 } & $\mathbf{1}$ & $\mathbf{2}$ & 0.133 \\
\hline Less informative & 0.622 & -0.127 & 0.039 \\
Not suiting personal needs & 0.808 & -0.128 & 0.171 \\
Relayed at the wrong time & 0.764 & -0.050 & 0.284 \\
Clutter as a result of too many ads & 0.608 & 0.021 & 0.463 \\
Cause disturbance at work & 0.537 & -0.094 & 0.650 \\
Busy work schedule & 0.295 & -0.158 & 0.695 \\
Wastage of time & 0.344 & -0.101 & -0.141 \\
Recall of brands advertised & -0.244 & 0.656 & -0.066 \\
Recall of sale/special promotions & -0.113 & 0.694 & -0.045 \\
Recall of products/services advertised & -0.096 & 0.779 & 0.082 \\
Ad positioning & -0.003 & 0.615 & -0.107 \\
Positive impact of mobile ads & 0.045 & 0.672 & 0.773 \\
Loss of privacy & 0.030 & 0.033 & \\
\hline
\end{tabular}


and loss of privacy. Since all these components are related to disturbance caused due to mobile advertising, this factor can be labelled 'Disruptive Nature' of mobile ads.

\section{Impact of user characteristics on perceptions about mobile marketing and buy/brand decision}

\section{$\mathbf{H}_{\mathbf{0 1}}$ : People of all age groups carry same perceptions about mobile marketing and the impact of mobile marketing on the buy/brand decision is also same for all age groups.}

There was found to be no age correlation in the perception of mobile advertising $(p>0.05)$, whereas there was found to be a correlation between age and buy/brand decision $(p<0.01)$. The reason for this could be that people of all age groups held the same perception about mobile marketing, but the impact of mobile marketing on buy/brand decision was found to be dependent upon age.

It was found that as age increases, features like location, contextualisation, relevance, and timing become more important (Figure 1), while the younger age groups

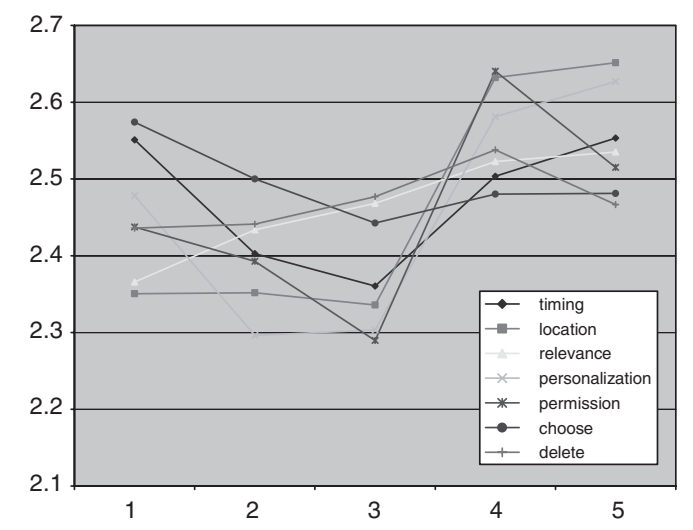

Figure 1: Perception map for age group and preferred features. Degree of requirement for a particular feature (timing, location, relevance, personalisation, permission, choice, and self-deletion) on a scale of $1-5$ on the $x$-axis, mean of age along the $y$-axis

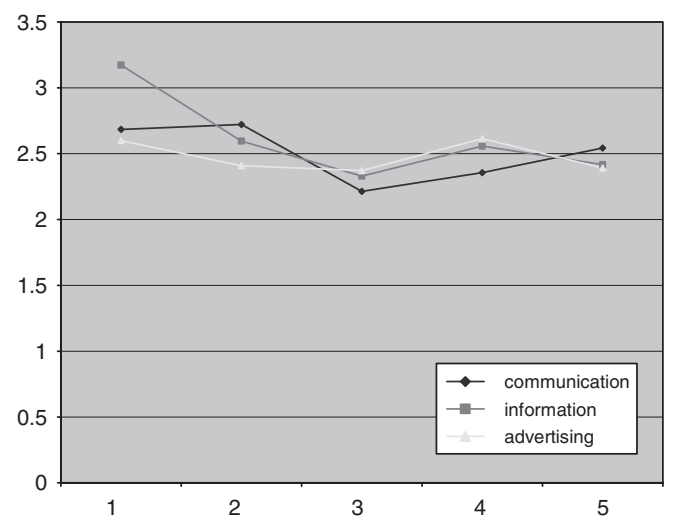

Figure 2: Perception map for age and purpose of mobile. Degree of usage of mobile for a particular purpose (communication, information, and advertising) on a scale of $1-5$ on the $x$-axis, mean of age along the $y$-axis

preferred features like choice and timing. The younger age groups, being students, felt the need to explore and use the mobile as a medium to gather more information. This corroborates with our finding that the younger age groups perceive the mobile as a device for information (Figure 2).

Marketers should use different models for mobile marketing for different age groups, where features like choice and interactiveness should be given preference for younger age groups, and features like relevance and contextualisation should be given preference for older age groups. A 'one size fits all' style of mobile marketing is not very effective.

$\mathbf{H}_{\mathbf{0 2}}$ : The impact of mobile marketing on buy/brand decision is dependent upon the individual work schedules and also the degree of impact is dependent upon individual work schedules.

There was found to be no relationship between impact of mobile marketing on buy/brand decision and individual work schedules $(p>0.05)$. However, there was found to be a significant relationship between the individual work schedules and 


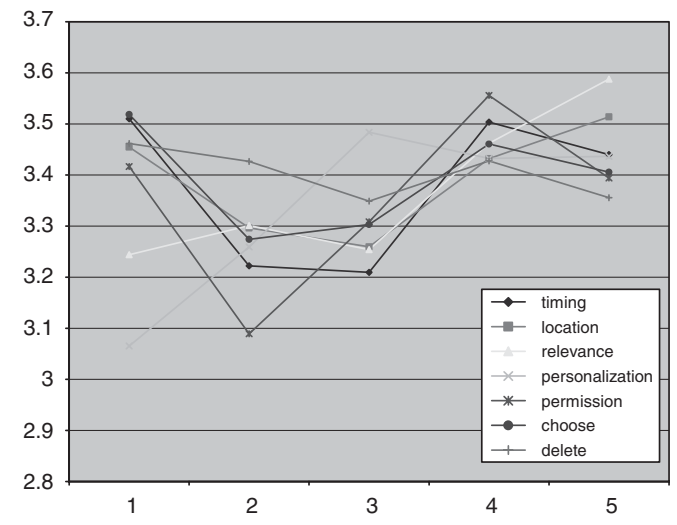

Figure 3: Perception map for work schedule and preferred features. Degree of requirement for a particular feature (timing, location, relevance, personalisation, permission, choice, and self-deletion) on a scale of $1-5$ on the $x$-axis, mean of work schedule busyness along the $y$-axis

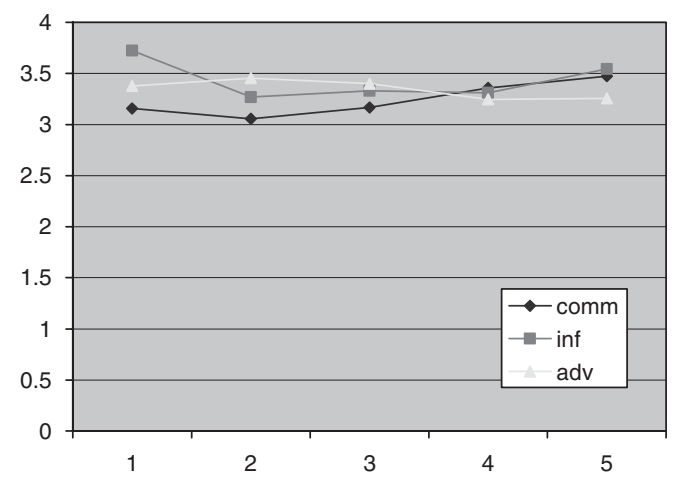

Figure 4: Perception map for work schedule and purpose of mobile. Degree of usage of mobile for a particular purpose (communication, information, and advertising) on a scale of $1-5$ on the $x$-axis, mean of work schedule busyness along the $y$-axis

the degree of impact (positive/negative) on perception about mobile marketing $(p<0.01)$ (Figures 3 and 4).

$\mathbf{H}_{03}$ : The perception of mobile marketing is independent of extent of mobile usage and the impact of mobile marketing on buy/brand decision is also independent of mobile usage.

It was found that perception of mobile marketing is independent of the extent of usage of the mobile $(p>0.05)$. Also, it was found that the impact of mobile marketing on buy decision was related to extent of

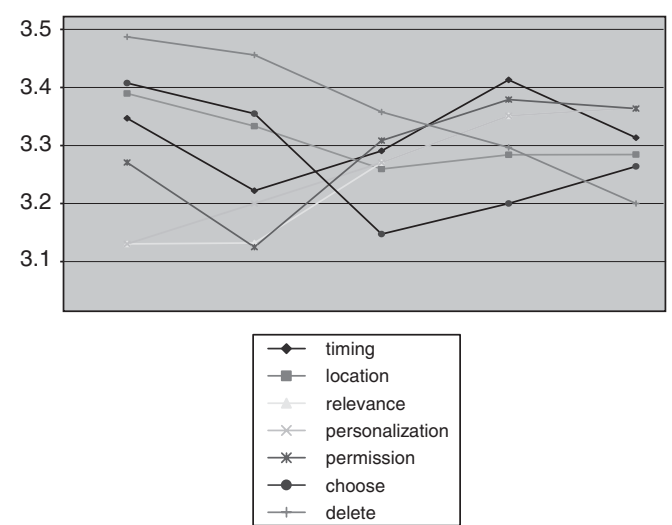

Figure 5: Perception map for extent of mobile usage and preferred features. Degree of requirement for a particular feature (timing, location, relevance, personalisation, permission, choice, and self deletion) on a scale of 1-5 on the $x$-axis, mean of mobile usage along the $Y$-axis

mobile usage $(p<0.05)$, but the brand decision was found to be independent of the extent of mobile usage $(p>0.05)$. At the same time, it was also found that the people whose extent of usage of mobile was low preferred features like browsing and choosing, while people whose extent of usage of mobile was high preferred features like contextualisation, personalisation, and relevance. The impact of mobile marketing could be more if the marketers positioned contextualisation and relevance of the ads to customer characteristics for heavy mobile users (Figure 5).

\section{DISCUSSION AND CONCLUSION}

The study shows that the perception people have about mobile advertising can be divided into three major factors: lack of contextualisation, perceived usefulness, and disruptive nature of mobile ads. The mobile was found to be a private medium of communication and thus mobile advertising was found to be intrusive. It might help to make mobile advertising more permissionbased and more contextualised so that the consumers perceive mobile advertising to be more useful and less disruptive.

To date, mobile marketing in India has been done in a one size fits all strategy. In 
the current scenario, the mobile marketers have not been able to reap the advantages afforded by the mobile as a medium of marketing. The study reports major gaps in the perception of mobile marketing and the benefits of the mobile as a medium of marketing communication. The major perceptions of mobile marketing have been found to be: the disruptive nature of mobile marketing, perceived usefulness of mobile marketing, and contextualisation of mobile marketing. The investigation also revealed that these perceptions have a significant impact on the customers buy/brand decision. Thus, it is important for marketers to address the issue of customer acceptance of mobile marketing and design the mobile marketing campaign in a manner that it is less intrusive and more useful and relevant to individual customer preferences. This can be done by using software agents to implement ontextualisation, timeliness, and relevance, which would lead to recall and increase the acceptance of the mobile as a medium of marketing.

It was also found that the younger age groups looked upon the mobile as a device of information, while the elder age groups looked upon the mobile as a device of communication. Thus, an ad campaign could be designed differently for different age groups, with features like interactivity and choice for the younger age groups and features like relevance and contextualisation for the elder age groups. Mobile marketing could be made more acceptable and effective in this manner.

\section{References}

1 Jelassi, T. and Enders, A. (2004) 'Leveraging wireless technology for mobile advertising', Proceedings of the 12th European Conference on Information Systems, Turku, Finland, 14-16 June.
2 Stewart, D. and Pavlou, P. (2002) 'From consumer response to active consumer: Measuring the effectiveness of interactive media', Journal of the Academy of Marketing Science, Vol. 30, No. 4, pp. 376-396.

3 Barnes, S. J. and Scornavacca, E. (2004) 'Mobile marketing: The role of permission and acceptance', International Journal of Mobile Communications, Vol. 2, No. 2, pp. 128-139.

4 Heinonen, K. and Strandvik, T. (2003) 'Consumer responsiveness to marketing communication in digital channels', in: Hannula, M., Järvelin, A-M. and Seppä, M. (eds.) 'FeBR2002 Frontiers of e-Business Research 2002', Tampere University of Technology and University of Tampere, pp. 137-152.

5 Windham, L. and Ken, O. (2002) 'The Soul of the New Consumer: The Attitudes, Behavior, and Preferences of E-Customers', Allworth Press, New York.

6 Li, H., Steven, M. E. and Lee, J. -H. (2002) 'Measuring the intrusiveness of advertisements: Scale development and validation', Journal of Advertising, Vol. 31, No. 2, 37-47.

7 Abernethy, A. M. (1991) 'Physical and mechanical avoidance of television commercials: An exploratory study of zipping, zapping and leaving', in: Holman, R. (ed.) 'Proceedings of the American Academy of Advertising', American Academy of Advertising, New York, pp. 223-231.

8 Marinova, M. and Massey (2002) 'Permission email marketing as a means of targeted promotion', Cornell Hotel and Restaurant Administration Quarterly, Vol. 43, No. 1, pp. 61-69.

9 Barwise, P. and Strong, P. (2002) 'Permission-based mobile advertising', Journal of Interactive Marketing, Vol. 16, No. 1, pp. 14-24.

10 Dickinger, A., Haghirian, P., Murphy, J. and Scharl, A. (2004) 'An investigation and conceptual model of SMS marketing', Proceedings of the 37th Hawaii International Conference on System Sciences, Big Island, Hawaii, January.

11 Kenny, D. and Marshall, J. F. (2000) 'Contextual marketing: The real business of the internet', Harvard Business Review, Vol. 78, No. 6, pp. 119-125.

12 Yuan, S. T. and Tsao, Y. W. (2003) 'A recommendation mechanism for contextualized mobile advertising', Expert Systems with Applications, Vol. 24, pp. 399-414.

13 Barnes, S. J. (2002) 'Wireless digital advertising: Nature and implications', International Journal of Advertising, Vol. 21, pp. 399-419.

14 Ververidis, C. and Polyzos, G. (2002) 'Mobile marketing using location based services', Proceedings of the First International Conference on Mobile Business, Athens, Greece. 